\title{
The Effect of Periocular Acupressure with a Medical Massager for Myopia Children
}

\author{
Ho-Yeol Cha ${ }^{1}$, A-Ram Jung ${ }^{1}$, Byoung-kab Kang ${ }^{2}$, Ji-Hoon Song ${ }^{3}$, Jae Ho Jung ${ }^{4}$, \\ Jin Hong Cheon ${ }^{1,3}$, Jun Yong Choi ${ }^{5}$, Kibong Kim, ${ }^{1,3, \#}$ \\ ${ }^{1}$ Department of Korean Pediatrics, Hospital of Korean Medicine, Pusan National University \\ ${ }^{2}$ KM Fundamental Research Division, Korea Institute of Oriental Medicine \\ ${ }^{3}$ Department of Korean Pediatrics, School of Korean Medicine, Pusan National University \\ ${ }^{4}$ Department of Ophthalmology, Pusan National University Yangsan Hospital, Pusan National University \\ ${ }^{5}$ Department of Internal Medicine of Korean Medicine, Hospital of Korean Medicine, Pusan National University
}

Objectives: Considering high prevalence of myopia in Asian countries, social cost paid for myopia may gradually increase in Korea. However, studies for developing myopia treatment are deficient. The study was to evaluate whether the periocular acupressure with a medical massager is effective for suppressing myopia progression.

Methods: This study was an investigator-sponsored, prospective, open-labeled, and superiority pre and post single-armed study. 14 myopia children aged 7-12 years wore a medical massager for 15 minutes to stimulate periocular acupoints. For 24 weeks, the participants used the device twice a day. Based on prior studies, the refraction change of naturally proceeded myopia was set as $-0.38 \mathrm{D}$. The axial length change of naturally proceeded myopia was set as $0.228 \mathrm{~mm}$. To assess the safety, we performed vital sign check, physical examination, visual acuity test, slit lamp examination, IOP measurement, and fundus examination.

Results: The refraction and axial length of the participants increased. For 24 weeks, the change in refraction was -0.38 $\mathrm{D}$ at the right eye and $-0.40 \mathrm{D}$ at the left. The change in axial length was $0.21 \mathrm{~mm}$ at the right and $0.22 \mathrm{~mm}$ at the left. In the safety assessment, any adverse event did not occur.

Conclusions: There was no significant difference between the control group and the intervention group. The effect of growth may have more contributed to our result than that of acupressure. Based on our result, a full-scale study will not be conducted.

$\overline{\text { Key Words }}$ : Myopia, Massage, Acupressure, Periocular, Medical device, Acupoint stimulation.

\section{Introduction}

In case of myopia, Asian countries including Korea, Taiwan, and Japan, which have higher enthusiasm for education, have a higher prevalence rate of $50-80 \%$ than that of $20-50 \%$ in USA and Europe $^{1)}$. Recently, the prevalence rate of myopia has been increasing. According to the Korea
National Health \& Nutrition Examination Survey in $2008,53 \%$ of the population older than 5 years old and $80.4 \%$ of the population at the age of $12-18$ years old have myopia ${ }^{2)}$. In many cases, this kind of myopia is called school myopia or growth-induced myopia because of near work at school. School myopia accounts for most of myopia.

Considering that Asian countries have higher

\footnotetext{
- Received:3 April 2016 • Revised : 22 June 2016 Accepted : 22 June 2016

- Correspondence to: Kim, Kibong

Department of Korean Pediatrics, Hospital of Korean Medicine, Pusan National University

20 Geumo-ro, Mulgeum-eup, Yangsan-si, Gyeongsangnam-do, 50612, Rep. of Korea.

Tel : +82-10-5026-2630, Fax : +82-55-360-5952, E-mail : kkb2630@gmail.com
} 
prevalence rate, Korean society may be going to pay much social cost for myopia in the future. Thus, the study for preventing and treating myopia progress may contribute to suppressing medical expenses increase by excluding unnecessary treatment.

There have been many efforts to develop treatments for preventing the progress of myopia: medications such as pirenzepine, tropicamide, and atropine, contact lenses, or special glasses such as overcorrection glasses, bifocal glasses, and progressive multifocal glasses $^{3)}$. However, the effective medication treatment for controlling myopia progression has not yet been developed. Glasses or contact lenses account for most of vision correction. Surgery such as keratotomy is the only way to change refraction or optic axis. However, surgery can be performed only in adults whose refractive error progression stopped. Surgery can also cause complications such as irregular astigmatism, glare at night, central islands, or corneal opacity.

Developing myopia treatment is necessary but relevant studies are deficient. There were several studies on the effect of acupressure for myopia. $\mathrm{Li}$ reported that the improvement rate of visual acuity was $92.24 \%$ in 1216 myopia patients ${ }^{4}$. Liu reported that the effective rate was $98.5 \%$ of 424 patients ${ }^{5}$. The effective ratio was $88.5 \%$ of 629 patients in Cheng and Chu's study ${ }^{6}$. From these studies, acupressure may be effective for myopia. However, these studies only evaluated the improvement in visual acuity and the subjective symptoms of the patient; they did not evaluate objective endpoints such as the change in refraction or axial length.

The objective of this study was to evaluate whether the acupressure on periocular acupoints with a massager is effective for suppressing the progression of myopia.

\section{Methods}

\section{Study Period}

This study was conducted from the approved date by the Korean Food and Drug Administration (KFDA) until December 31st, 2014.

\section{Study Design}

We designed this study as a pilot trial to suggest a Korean medical therapy for myopia. This study was an investigator-sponsored, prospective, and superiority pre and post single-armed study. This study was conducted as an open-label trial that does not blind both the investigators and participants.

We used a medical massager to evaluate the efficacy of periocular acupressure for myopia. After 24 weeks, we analyzed whether periocular acupressure effectively suppresses myopia progression comparing with the baseline.

\section{Ethics}

This study obeyed the Declaration of Helsinki. This study was approved by the Institutional Review Board (IRB) of Pusan National University Korean Medicine Hospital (PNUKH) (approval No.: 2013022). This study was enrolled in the Clinical Research Information Service (CRIS; http://cris.nih.go.kr) (CRIS Registration Number: KCT0001248) and ClinicalTrials. gov (http://www.clinicaltrials.gov) (ClinicalTrials.gov Identifier: NCT02064660). Prior to starting the trial, we received written consent from all participants.

\section{Participants Recruitment}

Recruitment was conducted at the PNUKH. The participant who passed screening tests(Table 1) and his/her guardian visited the center to receive instructions for this study such as the massager, warnings, and specific requirements. After the 1st visit, the trial was conducted at each participant's home.

At first, 37 children applied to our study. In the screening phase, 17 children were dropped out; 6 children were anisometropia, 5 children were 
Table 1. Inclusion and exclusion criteria

\begin{tabular}{ll}
\hline Criteria \\
\hline Inclusion & Children between 7-12 years old \\
& Children who have spherical equivalent less than -5 D and were diagnosed with school myopia \\
& No strabismus noted by covered tests at near and far gaze \\
& No anisometropia (The difference between spherical equivalents of each eye should be within 1.0 D.) \\
& Participation should be determined voluntarily or by children's guardian and participants should \\
& sign a written consent \\
\hline Exclusion & Glaucoma, cataract, retinal detachment or other severe eye disease \\
& Eye or head injury \\
& Children who had an operation at the eyes and then the surgical site has not yet healed \\
& Children who have a high fever or have a difficulty with wearing or operating the device alone \\
& Children who are currently wearing bifocal lenses or had worn them \\
& Systemic or neurological problems that affect vision development \\
& Long-time medication that affects visual acuity or myopia progress
\end{tabular}

hyperopia, 3 children were high myopia, 2 children withdrew consent because of discomfort caused by tests, and 1 child withdrew consent because of test refusal. Thus, the trial started with 20 children. Moreover, 6 children was dropped out during the trial; 2 children had difficulty in visiting center because of school hour, 2 children were stopped by their guardians because they did not wear the device steadily, 1 child was out of contact on the scheduled visit date, and 1 children had difficulty in and avoided wearing the device. As a result, 14 children successfully finished the trial.

\section{Intervention}

\section{1) Medical Device}

This study used a medical massager (Nurieye-1, Seodong Medical Co. South Korea) which stimulates periocular area with vibration. The periocular acupoints such as BL1, ST1, BL2, GB37, KI5, EX-HN14, Xià-jīngmíng (下睛明), and GB16 have been mainly used as the main acupoints for myopia. The device has 22 acupressure rods which only touch the periocular area. When vibration was delivered to the touched area, the device stimulates the area automatically. The device consists of an exterior case, a control panel, acupressure rods made of soft silicon and jade tip, and an adaptor. Fig. 1 is the image of the device.

Participants took an acupressure treatment on the periocular acupoints with the massager. For 24 weeks, participants used the device for 15 minutes twice a day.

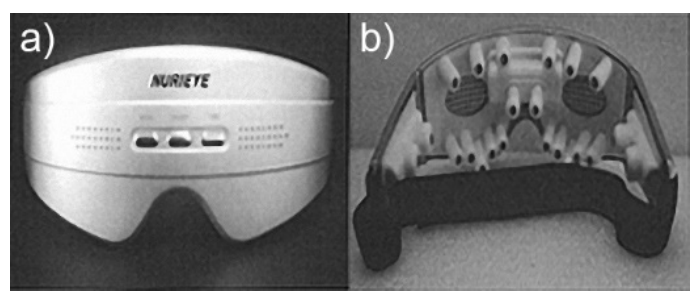

Fig 1. Nurieye-1, a medical massager used in this study. a): an anterior view; b): a posterior view.

2) Study Procedure

At the 1st visit, we conducted interview, ophthalmological examination, and adverse event assessment. The massager was also distributed to participants at that time. The participants visited the center after 12 and 24 weeks. Ophthalmological tests, adverse event assessment, and compliance assessment was conducted at the 2 nd and 3rd visit.

\section{(1) Applicant Screening}


An investigator had an interview with applicants to investigate baseline characteristics, medical history, and whether or not to include. The inclusion and exclusion criteria were as Table 1. Suitable applicants were received the participant identification code.

(2) Ophthalmological Examinations

Consented participants took ophthalmological tests. A pediatric ophthalmologist who has more than 3 years of experience conducted ophthalmologic examinations including visual acuity test, slit lamp examination, refraction inspection, intraocular pressure (IOP) measurement, and fundus examination. Tests were performed by the same experienced examiner at every visit. To minimize the change in participants' behavior that affects myopia, all participants were forbidden from knowing their initial spherical equivalent. The main endpoints were the change in refraction and axial length for 24 weeks. Visual acuity test, slit lamp examination, IOP measurement, and fundus examination were performed to assess the safety of the device.

\section{(1) Refraction Inspection}

Refraction change for 24 weeks was the primary endpoint in this study. As an untreated group, we used historical control group. According to prior studies on school myopia progression, the refraction change of naturally proceeded myopia was $-0.38 \mathrm{D}$ for 6 months in pediatric myopia patients ${ }^{7,8)}$. Thus, we set the refraction change of the control group as -0.38 D.

We used cycloplegic refraction among various refraction tests. It is difficult to assess the exact refraction in children. Because children have very strong accommodation force, a children can be diagnosed as myopia even if he/she has hyperopia. Thus, it is an essential test for assessing refraction in children $^{9)}$. A full auto ref-keratometer (RK-F1 Full Auto Ref-keratometer, Canon Components, Inc.,
Japan) was used for the cycloplegic refraction. The test was conducted 1 hour after from applying tropicamide/phenylephrine hydrochloride mydriatics (Midrin-P ophthalmic solution, Santen Pharmaceutical Co., Ltd., Japan) ${ }^{10)}$. The mydriatics was applied 3 times at 5 -minute intervals ${ }^{10}$. Then we tested the accommodation with a penlight.

\section{(2) Axial Length Measurement}

Axial length change for 24 weeks was the secondary endpoint in this study. Based on prior studies on school myopia progression, the axial length change in the historical control group over 6 months was calculated as $0.228 \mathrm{~mm}^{11)}$. Thus, we set the axial length change of the control group as 0.228 $\mathrm{mm}$.

Physiologically, myopia is related to the refraction and the axial length; especially, the axial length greatly contributes to. Several studies reported that myopia progress is associated with the increase in axial length ${ }^{12,13}$. Axial length was measured with IOL Master (Carl Zeiss, Dublin, CA, USA). Axial length measurement prevents the interference of the examiner's bias which is arisen when the refraction inspection is only conducted. Consequently, measuring axial length makes identifying the degree of myopia exactly.

\section{(3) Visual Acuity Test}

Visual acuity test was performed with the Han Chun-suk visual acuity chart which is widely used. This test relatively easily detects the degree of myopia within 3 minutes ${ }^{14,}$ 15).

\section{(4) Slit Lamp Examination}

Slit lamp examination identifies the abnormality of the cornea and lens with a slit lamp microscope. Slit Lamp BQ-900 (Haag-Streit AG, Switzerland) was used in this study. With this test, we were able to identify adverse events or whether the participants meet the exclusion criteria. 


\section{(5) IOP Examination}

According to a prior study, the average IOP of the myopia group was significantly higher than that of the non-myopia group in children ${ }^{16)}$. Thus, we performed IOP examination in this study. A full auto tonometer (Canon Full Auto Tonometer TX-F, Canon Components, Inc., Japan) was used.

\section{(6) Fundus Examination}

Fundus examination helps to diagnose abnormalities of the retina, choroid, and optic nerve. By conducting fundoscopy at each visit, we were able to examine other ophthalmic diseases or adverse events. Non-mydriatic retinal camera (TRC-NW200, Topcon Corporation, Tokyo, Japan) was used.

\section{Statistical Analysis}

Primary and secondary endpoints were presented as the number of people, mean, and standard deviation (SD). After performing normality test using Shapiro-Wilk method, p-values of the change in the primary and secondary endpoint between the 1st and $3 \mathrm{rd}$ visit were calculated with one sample t-test or one sample Wilcoxon signed rank test. Two-tailed p-value less than 0.05 was considered to be statistically significant. The data were analyzed using the SPSS version 21.0 (SPSS Inc., Chicago, IL, USA).

\section{Results}

\section{Gender and Age}

At the 1st visit, we investigated basic information of the participants. Among 37 applicants, total 14 children successfully finished the trial. The gender of the participants was 5 males and 9 females. The average age was $8.93 \pm 1.33$ years.

\section{Efficacy Evaluation}

Wearing the massager did not suppress the increase in both refraction and axial length. In refraction, the mean refraction of the right eye was $-2.76 \mathrm{D}$ and that of the left eye was $-2.85 \mathrm{D}$ at the 1st visit. After 24 weeks, the mean refraction of the right eye was $-3.13 \mathrm{D}$ and that of the left eye was $-3.25 \mathrm{D}$. In axial length, the mean axial length of the right eye was $24.41 \mathrm{~mm}$ and that of the left eye was $24.47 \mathrm{~mm}$ at the 1st visit. After 24 weeks, the mean axial length of the right eye was $24.63 \mathrm{~mm}$ and that of the left eye was $24.69 \mathrm{~mm}$ (Table 2).

For 24 weeks, the refraction had changed as much as $-0.38 \mathrm{D}$ at the right and $-0.40 \mathrm{D}$ at the left. The axial length had changed as much as $0.21 \mathrm{~mm}$ at the right and $0.22 \mathrm{~mm}$ at the left. P-values of the change in refraction and axial length were statistically significant (Table 3). For 24 weeks, refraction and axial length changes of control group is $-0.38 \mathrm{D}$ and $0.228 \mathrm{~mm}$ respectively. Thus, there were no significant differences between control group and periocular acupressure group in the refraction and axial length.

\section{Safety Assessment}

To assess the safety of the device and adverse events, we performed vital sign check, physical examination, visual acuity test, slit lamp examination, IOP measurement, and fundus examination at each visit. As a result, any adverse event threatening children did not occur.

\section{Discussion}

This study was a pilot trial to evaluate the efficacy of periocular acupressure for myopia in children. A medical massager was used to stimulate periocular acupoints. We measured the refraction and axial length at 12th and 24th week. Then, we compared the change in refraction and axial length for 24 weeks with the results of prior studies.

Atropine, pirenzepine, and contact lenses partly suppress myopia progress, however, there is no 
Table 2. The refraction and axial length at each visit

\begin{tabular}{cccccccccccc}
\hline & & \multicolumn{3}{c}{ V1 (first visit) } & \multicolumn{3}{c}{ V2 (12th week) } & \multicolumn{3}{c}{ V3 (24th week) } \\
\cline { 3 - 11 } & & $\mathrm{n}$ & Mean & SD & $\mathrm{n}$ & Mean & SD & $\mathrm{n}$ & Mean & SD \\
\hline \multirow{2}{*}{ Refraction (D) } & $\mathrm{Rt}$ & 14 & -2.76 & 1.22 & 14 & -3.04 & 1.16 & 14 & -3.13 & 1.24 \\
& $\mathrm{Lt}$ & 14 & -2.85 & 1.28 & 14 & -3.1 & 1.32 & 14 & -3.25 & 1.21 \\
\multirow{2}{*}{ Axial Length (mm) } & $\mathrm{Rt}$ & 14 & 24.41 & 0.66 & 14 & 24.52 & 0.64 & 14 & 24.63 & 0.62 \\
& $\mathrm{Lt}$ & 14 & 24.47 & 0.72 & 14 & 24.58 & 0.71 & 14 & 24.69 & 0.68 \\
\hline
\end{tabular}

Abbreviations: $\mathrm{n}$ : number of participants; SD: standard deviation; Rt: right eye; Lt: left eye.

Table 3. The change in refraction and axial length for 24 weeks

\begin{tabular}{|c|c|c|c|c|c|}
\hline & & $\mathrm{n}$ & Mean & $\mathrm{SD}$ & P-value \\
\hline \multirow{2}{*}{ Refraction Change (V3-V1) (D) } & $\mathrm{Rt}$ & 14 & -0.38 & 0.33 & $0.0009^{*}$ \\
\hline & $\mathrm{Lt}$ & 14 & -0.40 & 0.26 & $<.0001^{*}$ \\
\hline \multirow{2}{*}{ Axial Length Change (V3-V1) (mm) } & $\mathrm{Rt}$ & 14 & 0.21 & 0.09 & $<.0001^{*}$ \\
\hline & $\mathrm{Lt}$ & 14 & 0.22 & 0.1 & $<.0001^{*}$ \\
\hline
\end{tabular}

*: statistically significant $(P<0.05)$.

Abbreviations: n: number of participants; SD: standard deviation; Rt: right eye; Lt: left eye.

fundamental treatment for school myopia ${ }^{17}$. Although its outstanding effect has been demonstrated by some studies, atropine is considered to be unacceptable for long-term therapy by many clinicians because of the side effects such as photophobia and cycloplegia ${ }^{18)}$. Single vision lenses, which are commonly prescribed spectacle lens to myopia children, may promote myopia progression and increase axial length ${ }^{18)}$. Bifocal and progressive lenses have been produced small and clinically insignificant therapeutic effects except children who are esophoric and have large accommodation lag $^{19}$ ). Traditional correcting soft and gas-permeable contact lenses have not been proved to be effective for suppressing myopia progression ${ }^{19)}$. Surgeries including laser-assisted in-situ keratomileusis (LASIK) and laser assisted sub-epithelial keratomileusis (LASEK), which are used to adults, are not suitable for school myopia ${ }^{17}$.

Korean medicine considers the cause of myopia as qi deficiency or excessive blood. For treatment, acupuncture, medicine, or exercise for eye has been used. In the acupuncture or acupressure treatment, six yang meridian and periocular acupoints were mainly chosen. It is hypothesized that the Chinese eye exercises, which are a kind of periocular acupressure and are originated from traditional Chinese medicine, increase blood flow to the eyeball, enhance parasympathetic-driven responses in the ciliary muscle by stimulating ocular region or visual cortex, and thereby effect the accommodation of the eyes ${ }^{20)}$. Massage on BL2, GB14, TE23, EX-HN1, ST1, GB20, LI4, LI11 and GV23 increase chorioretinal $^{21)}$ and regional cerebral blood flow ${ }^{22}$. Massage on BL2 and ST1 promotes tear stability and secretion, increases tear lactoferrin level, and affects retrobulbar circulation and $\mathrm{IOP}^{23)}$.

Prior studies including $\mathrm{Li}^{4}$, $\mathrm{Liu}^{5}$, Cheng and $\mathrm{Chu}^{6)}$, and $\mathrm{Tao}^{24)}$ has been showed that periocular acupuncture or acupressure have an effect for improving myopia. However, there have been no acupuncture or acupressure study for myopia which used objective endpoint such as change in refraction. Thus, we set the refraction change, which is clinically used and objective, as the primary endpoint to measure accurate visual acuity,

We did not set the control group in this study. Generally, setting a control group increases the reliability of the study. However, setting a control 
group can be skipped when primary endpoint is objective without biases ${ }^{25}$ or participants are vulnerable to being violated their right or risks ${ }^{26)}$. Prior studies on school myopia progression demonstrated that the changes in refraction and axial length of naturally proceeded myopia for 6 months were $-0.38 \mathrm{D}$ and $0.228 \mathrm{~mm}$, respectively ${ }^{7,8,11)}$. Thus, we set the change in refraction and axial length of the control group as $-0.38 \mathrm{D}$ and $0.228 \mathrm{~mm}$.

In this study, there was no remarkable effect on suppressing the change in refraction and axial length. For 24 weeks, the refraction increased as much as that of the control group, whose refraction change was $-0.38 \mathrm{D}$. The change in refraction was $-0.38 \mathrm{D}$ at the right and $-0.40 \mathrm{D}$ at the left. The axial length more increased than that of the control group, whose axial length change was $0.228 \mathrm{~mm}$. The change in axial length was $0.21 \mathrm{~mm}$ at the right and $0.22 \mathrm{~mm}$ at the left. In other words, there was no significant differences between control group and the intervention group in the refraction and axial length. For safety, there was no adverse events in the safety assessment. Thus, the massager is considered to safe for children.

Comparing with pseudomyopia which is caused by ciliary spasm, simple myopia is caused by refraction which is greater than axial length of the eye $^{27)}$. As the eye grows, the lens adds layers of tissue but becomes thin and flat by stretching in the equatorial plane to maintain emmetropia; if this process continues, the thinned lens is unable to compensate for the increasing axial length ${ }^{28)}$. As a result, the eye becomes myopic and the shape of eyeball becomes more prolate ${ }^{28)}$. The progress of simple myopia, which is the most of school myopia, begins at the age of 10, develops slowly, and ends at the age of $25^{29)}$. Thus, our result seems to be because the effect of eye growth was greater than that of periocular acupressure.

Several prior studies also have shown that eye exercises on acupoints or acupuncture are insufficient for preventing myopia progression. When children aged 10-14 years with emmetropia to moderate myopia performed Chinese eye exercises on periocular acupoints, there was no significant difference between before and after intervention in the findings of near and distant visual acuity, visual discomfort score and the proportions of improvement for these outcomes $^{20)}$. In another study on the Chinese eye exercises, near vision symptoms were modestly relieved among Chinese urban children aged 6-17 years but there were no remarkable effect on reducing myopia ${ }^{30)}$.

Similarly, any results from our study were not able to demonstrate that the periocular acupressure is remarkably effective for delaying myopia progression in children. Further studies on periocular acupressure for delaying myopia progression should be designed as a randomized controlled trial (RCT) with large sample size. If we follow up the axial length of the participants for a long time more than 1 year, we may obtain good results.

\section{Conclusion}

This study was a pilot study for evaluating whether the periocular acupressure is effective for suppressing myopia progression in children. For 24 weeks, 14 myopia children wore a medical massager to stimulate periocular acupoints. For untreated group, the historical control group was used.

As a result, there was no significant difference between the control group and the intervention group in the refraction and axial length. Our study was not able to demonstrate the remarkable benefit of periocular acupressure for delaying myopia progression in children. The effect of growth may have more contributed to our result than that of acupressure. Based on our result, a full-scale study will not be conducted. 


\section{Abbreviations}

KFDA: Korean Food \& Drug Administration; IRB: Institutional Review Board; PNUKH: Pusan National University Korean Hospital; CRIS: Clinical Research Information Service; IOP: intraocular pressure; SD: standard deviation; LASIK: laser-assisted in-situ keratomileusis; LASEK: laser-assisted sub-epithelial keratomileusis; RCT: randomized controlled trial.

\section{Acknowledgement}

This study was funded by a project grant from Korean Institute of Oriental Medicine (K16123).

\section{Competing Interests}

The authors declare that they have no competing interests.

\section{References}

1. Choi TH, Jung JW, Choi YY. The Effect of Atropine on Myopic Progression in Children. J Korean Ophthalmol Soc. 2005;46(7):1189-95.

2. Disease Prevention Center. 2008 National Health Statistics. Seoul:Centers for Disease Control and Prevention. 2009.

3. Saw SM, Gazzard G, Au Eong KG, Tan DTH. Myopia: attempts to arrest progression. $\mathrm{Br} \mathrm{J}$ Ophthalmol. 2002;86(11):1306-11.

4. Li Z. Observation the Therapeutical Effect of 1216 Cases of myopia with the treatment of ear-acu-pressure by Semen Vaccariae in combintion with qigong. Chinese Acupuncture and Moxibustion. 1991;11(1):7-8.

5. Liu H, Lü Y, Dong Q, Zhong X. Treatment of adolescent myopia by pressure plaster of semen impatientis on otoacupoints. J Tradit Chin Med. 1994;14(4):283-6.

6. Cheng P, Chu J. Treatment of 1673 myopic eyes by auricular on adolescent. Journal of Practical Traditional Chinese Internal Medicine. 2003;17(3):238.

7. Kim JR, Chung TY, Lim DH, Bae JH. Effect of orthokeratologic lenses on myopic progression in childhood. J Korean Ophthalmol Soc. 2013; 54(3):401-7.

8. Chua WH, Balakrishnan V, Chan YH, Tong L, Ling Y, Quah BL, et al. Atropine for the treatment of childhood myopia. Ophthalmology. 2006;113(12):2285-91.

9. Choi YH, Choi YY. The difference comparison according to child refractive method and effect of life style on myopia. J Korean Ophthalmol Soc. 2005;46(11):1841-7.

10. Shin KM, Chung SA, Lee JB. Comparative study on the efficacy of different cycloplegic agents in myopic adults. J Korean Ophthalmol Soc. 2011;52(2):141-6.

11. Chen M, Dai J, Chu R, Qian Y. The efficacy and safety of modified Snyder-Thompson posterior scleral reinforcement in extensive high myopia of Chinese children. Albrecht Von Graefes Arch Klin Exp Ophthalmol. 2013; 251(11):2633-8.

12. Kim HJ, Kim HA, Jeon IC, Kim SY, Jang MH, Mah KC. Downward deviation at reading in children wearing progressive addition lens. Korean J Vis Sci. 2013;15(4):405-15.

13. Shih YF, Hsiao CK, Chen CJ, Chang CW, Hung PT, Lin LL. An intervention trial on efficacy of atropine and multi-focal glasses in controlling myopic progression. Acta Ophthalmol Scand. 2001;79(3):233-6.

14. Baek SC, Lee DH, Kim SH, Lee SJ, Park SH. Relationship between uncorrected visual acuity and refractive error in visual acuity test of children. J Korean Ophthalmol Soc. 2004;45(6): 1001-8.

15. Yang HN, Koo BS. Inter-relationship between visual acuity and refractive error in myopia and 
hyperopia among children with decreased visual acuity. J Korean Ophthalmol Soc. 1985;26(5): $639-44$.

16. Kwon GR, Ahn CS. The correlation between intraocular pressure and myopia in children. $\mathrm{J}$ Korean Ophthalmol Soc. 1998;39(4):702-7.

17. Oh SY. Prevention and treatment of school myopia. J Korean Med Assoc. 2007;50(3): $259-64$.

18. Gwiazda J. Treatment options for myopia. Optom Vis Sci. 2009;86(6):624-8.

19. Cooper J, Schulman E, Jamal N. Current status on the development and treatment of myopia. Optometry. 2012;83(5):179-99.

20. Li SM, Kang MT, Peng XX, Li SY, Wang Y, Li $\mathrm{L}$, et al. Efficacy of Chinese eye exercises on reducing accommodative lag in school-aged children: a randomized controlled trial. PLoS ONE. 2015;10(3):e0117552.

21. Naruse S, Mori K, Kurihara M, Nakajima N, Matsumoto Y, Kinoshita S, et al. Chorioretinal blood flow changes following acupuncture between thumb and forefinger. Nippon Ganka Gakkai zasshi. 2000;104(10):717-23.

22. An YS, Moon SK, Min IK, Kim DY. Changes in regional cerebral blood flow and glucose metabolism following electroacupuncture at LI 4 and LI 11 in normal volunteers. J Altern Complement Med. 2009;15(10):1075-81.

23. Kim TH, Kang JW, Kim KH, Kang KW, Shin MS, Jung SY, et al. Acupuncture for the treatment of dry eye: a multicenter randomised controlled trial with active comparison intervention (artificial teardrops). PLoS One. 2012;7(5):e36638.

24. Tao XY, Sun CX, Yang JL, Mao M, Liao CC, Meng JG, et al. Clinical observation on strong stimulation of acupoints around eyes for treatment of adolescent myopia. Chinese acupuncture \& moxibustion. 2008;28(3):191-3.

25. An HJ. A research on statistical methods of clinical trials in medical device. Seoul:National Institute of Food and Drug Safety Evaluation. 2009.

26. Korea Food \& Drug Administration. Guidelines for Pediatric Clinical Evaluation. Seoul:2007.

27. Chang DJ, Joo CK. Current and future options for myopia correction. J Korean Med Assoc. 2012;55(4):362-70.

28. Myrowitz EH. Juvenile myopia progression, risk factors and interventions. Saudi J Ophthalmol. 2012;26(3):293-7.

29. Lee JH, Cho SE, Woo YM, Nam Y. A Clinical Study of Acupuncture Effect on Children's Myopia. J Korean Med Ophthalmol Otolaryngol Dermatol. 2001;14(2):253-61.

30. Lin Z, Vasudevan B, Jhanji V, Gao TY, Wang NL, Wang Q, et al. Eye exercises of acupoints: their impact on refractive error and visual symptoms in Chinese urban children. BMC Complement Altern Med. 2013;13:306. 